Institute for Computational Mathematics

Hong Kong Baptist University

ICM Research Report

09-07 


\title{
Collocation Methods for Hyperbolic Partial Dif- ferential Equations with Singular Sources
}

\author{
Jae-Hun Jung ${ }^{1 *}$, Wai Sun Don ${ }^{2}$ \\ 1 Department of Mathematics, the State University of New York at Buffalo, \\ Buffalo, NY 14260-2900 \\ 2 Department of Mathematics, Hong Kong Baptist University, Hong Kong, \\ China
}

\begin{abstract}
A numerical study is given on the spectral methods and the high order WENO finite difference scheme for the solution of linear and nonlinear hyperbolic partial differential equations with stationary and non-stationary singular sources. The singular source term is represented by the $\delta$-function. For the approximation of the $\delta$-function, the direct projection method is used that has been proposed in [6]. The $\delta$-function is constructed in a consistent way to the derivative operator. Nonlinear sine-Gordon equation with stationary singular source was solved with the Chebyshev collocation method. The $\delta$-function with the spectral method is highly oscillatory but yields good results with small number of collocation points. The results are compared with those computed by the second order finite difference method. In modeling general hyperbolic equations with a non-stationary singular source, however, the solution of the linear scalar wave equation with the non-stationary singular source using the direct projection method yields non-physical oscillations for both the spectral method and the WENO scheme. The numerical artifacts arising when the non-stationary singular source term is considered on the discrete grids are explained.
\end{abstract}

AMS subject classifications: 65M06, 65M30, 65M70

Key words: Singular sources, Dirac- $\delta$-function, Direct projection method, Chebyshev collocation method, WENO scheme.

\section{Introduction}

In various mathematical modeling of physical processes, such as the shock-particle laden flows [5] and the collisions of black-holes [8], the system of partial differential equations

\footnotetext{
${ }^{*}$ Corresponding author. Email addresses: jaehun@buffalo.edu (J.-H. Jung), wsdon@math.hkbu.edu.hk (W.-S. Don) 
(PDEs) often involves point sources as a forcing term on the right hand side of PDEs. For example, consider the following 1D scalar hyperbolic equation

$$
U_{t}+F_{x}(U)=S(x, t), \quad U: \mathbb{R} \times \mathbb{R}^{+} \rightarrow \mathbb{R}, \quad t>0,
$$

where $F(U)$ is the flux with real Jacobian $\partial F / \partial U$ and $S(x, t)$ is the source term consisting of a singular function such as the $\delta$-function and its derivative(s).

In this work, we consider some numerical issues related to the solution of PDEs with a stationary source $(S(x)$ is a function of space only) and a non-stationary singular source $(S(x, t)$ is a function of both space and time) with the collocation methods such as the spectral methods and high order weighted essentially non-oscillatory (WENO) finite difference methods (see $[1,3,4,13]$ and references contained therein for details of these methods). It should be noted that the WENO methods is particularly suitable for solving problem with solution containing discontinuities. Following the convention, the singular sources are represented by the Dirac- $\delta$-function and/or its derivative(s).

Due to the singular nature of the point sources, smooth initial condition may yield a solution containing discontinuity even for linear scalar equation. High order numerical approximations of such nonsmooth solutions would suffer the well-known Gibbs phenomenon $[3,4]$. Consequently, the numerical solutions become oscillatory near the singularity and high order convergence will be lost near the singularity. In time-dependent problem, the scheme would even become unstable. To handle this situation, the point sources are often regularized to obtain a smoother representation of the $\delta$-function such that the approximation converges to the $\delta$-function in the usual limit sense. Several related regularization methods have been developed $[2,11,12,14]$. The Gaussian function approximation (GA) method is the simplest approximation popularly used. One can also make use of an alternative definition of the $\delta$-function that the derivative of the Heavside function $H(x)$ is the delta function in the distribution sense, namely, $d H(x) / d x=\delta(x)$. The derivative operator will then be incorporated into the derivative operator of the flux function $F$. This is what we called the Direct Projection (DP) method in this study which was proposed in [6].

In this paper, we focus our discussion on the numerical approximations of the PDEs on a set of discrete grid points and we shall limit our discussion to the one-dimensional scalar linear and nonlinear problems. We will demonstrate the application of the DP method for solving the nonlinear sine-Gordon equation with a stationary singular source term using the Chebyshev collocation method [3,4,6] and compare the results obtained by the second order finite difference scheme using the GA method. The solution with the spectral method converges quickly when the number of grid points is increased.

For a non-stationary singular source that is moving in time, one have to consider the effect of the movement of the singular source in time and space. The $\delta$-function can be located between two discrete grid points causing errors in modeling of the $\delta$-function. We demonstrate, through a simple linear scalar wave equation with a non-stationary singular source, that numerical oscillations could be generated due to the movement of the $\delta$ function through the discrete grids as the location of the $\delta$-function is no longer located 
exactly at the grid point at a given time. The frequency or wavelength of the oscillations is directly related to the product of the number of the grid points and the speed of the propagation of the $\delta$-function.

The paper is composed of the following sessions. In Section 2, we briefly describe two approximation methods for the singular source term, namely, the GA method and the DP method for linear and nonlinear scalar PDEs. In Section 3, we consider scalar PDEs with stationary singular source term. We solve the linear scalar wave equation with stationary singular source by the Chebyshev collocation method and the fifth order WENO finite difference scheme while the singular source term is approximated by the DP method. We also solve the nonlinear sine-Gordon equation with a stationary singular source. The numerical experiments based on the Chebyshev collocation method with the DP method and the second order finite difference method with the GA method for the singular source are presented. We present some results of the sine-Gordon problem at the sub-critical and super-critical velocities. In Section 4, we illustrate the non-physical oscillations generated by a non-stationary singular source on a finite grid. The linear scalar wave equation with a non-stationary singular source is served as a model to demonstrate the phenomenon. The PDEs is solved by the high order WENO-Z finite difference scheme while the singular source is approximated by the DP and GA methods. A brief summary and future research direction is given in Section 5 .

\section{Approximations of singular sources}

In this study, we consider the equation (1.1), the scalar hyperbolic equation with a singular source (forcing) term, $S(x, t)=S(x+c t)$. For simplicity, we consider the case that the propagating speed $c$ of the singular source term is either zero or a real constant for all time $t$.

Let $S$ be represented by the Dirac $\delta$-function and/or its derivative(s). The Dirac $\delta$ function and its derivatives are defined by the following equations in the distribution sense, i.e., for a given real-valued function $f(x)$ in the domain $\Omega=[-1,1]$

$$
\int_{-1}^{1} f(x) \delta(x-y) d x=f(y)
$$

and

$$
\int_{-1}^{1} f(x) \delta^{\prime}(x-y) d x=-f^{\prime}(y), \quad \int_{-1}^{1} f(x) \delta^{\prime \prime}(x-y) d x=f^{\prime \prime}(y), \quad \ldots
$$

where $-1<y<1$ and the superscript ' denotes the derivative with respect to $x$.

Since the Dirac $\delta$-function is singular in nature and only defined in the distribution sense, it is not possible to calculate its value directly. In order to model the $\delta$-function, we consider the following two possible cases; 
- approximate the $\delta$-function using the GA method as

$$
\delta_{G}(x-a)=\frac{1}{\sigma \sqrt{\pi}} \exp \left(-\frac{(x-a)^{2}}{\sigma^{2}}\right),
$$

where $\sigma$ is chosen such that $\delta_{G}$ is sufficiently small near $x= \pm 1$. On a given grid with $N+1$ collocation points, $\sigma$ also decays with $N$ such that $\sigma=\sigma(N)=\frac{\alpha_{0}}{N}$. The free parameter $\alpha_{0}$ is chosen such that the integral value of $\delta_{G}$ over $[-1,1]$ converges to 1 based on the quadrature if $N \rightarrow \infty$.

- approximate the $\delta$-function using the DP method, i.e.,

$$
\delta_{N}(x-a)=D_{N} H_{N}^{a}(x),
$$

where $D_{N}$ is the derivative operator for a certain numerical method such as the finite difference method on a prescribed set of $N+1$ grid points, the spectral collocation method of a given type of Gauss-quadrature points, or the WENO method. And the $\delta_{N}(x-a)$ and $H_{N}^{a}(x)$ are the approximation of the $\delta$-function and the Heaviside function defined on the given set of collocation points.

The rationality of the above definition of the $\delta$-function for the DP method is that

$$
\delta(x-a)=\frac{d}{d x} H^{a}(x),
$$

where $H^{a}(x)$ is defined by

$$
H^{a}(x)=\left\{\begin{array}{cc}
0 & x<a \\
\frac{1}{2} & x=a \\
1 & x>a
\end{array} .\right.
$$

If the $\delta$-function exists between the grid point such that $x_{j}<a<x_{j+1}$ for a certain grid index $j$, the discrete Heaviside function $H_{N}^{a}(x)$ is given by

$$
H_{N}^{a}\left(x_{j}\right)=\left\{\begin{array}{cc}
0 & x_{j}<a \\
1 & x_{j}>a
\end{array} .\right.
$$

In this work, we employed the derivative operator $D_{N}$ for the spectral method with the Chebyshev-Gauss-Lobatto collocation points, $x_{j}=\cos (\pi j / N), \quad j=0, \ldots, N$, where $N+1$ is the total number of collocation points. For the WENO finite difference scheme, we define the derivative operator in a consistent way to the WENO derivative operation.

\section{PDEs with a stationary singular source}

In [6], several equations have been used to demonstrate the accuracy and efficiency of the DP method mostly for the linear problems. In this section, we present numerical results 
for both linear and nonlinear scalar PDEs with a stationary singular source term. For the linear scalar PDEs, we solve the linear wave equation with a stationary singular source by the Chebyshev collocation method and the fifth order WENO finite difference method. For the nonlinear scalar sine-Gordon equation, we solve the PDEs with both the Chebyshev collocation method with the DP method and the second order finite difference method with the GA method.

\subsection{Linear wave equation}

Consider the scalar wave equation with a stationary singular source term in the domain $x \in[-1,1]$,

$$
\begin{aligned}
u_{t}+u_{x} & =\delta(x), \quad x \in[-1,1], \quad t>0 \\
u(x, 0) & =\sin (\pi x), \\
u(-1, t) & =\sin (\pi(-1-t)),
\end{aligned}
$$

where a stationary singular source term is located at $x=0$. The exact solution of the problem above is $u(x, t)=\sin (\pi(x-t))+H(x)$.

Using the Chebyshev collocation method for the spatial differential term and the DP method for the singular source term, the semi-discrete formulation of the equation (3.1) can be expressed as

$$
\frac{d}{d t} U_{j}(t)=-\left.D_{N}\left(U(x, t)-H_{N}(x)\right)\right|_{x=x_{j}}
$$

where $H_{N}(x)$ is the discrete Heaviside function with jump at $x=0$ and $U(t)$ is the solution vector defined on the grid points $x_{j}$. The system of ODE can now be advanced in time by a third order Runge-Kutta scheme. In [6], it has been shown that the DP method yields spectral accuracy for the linear wave equation (Figure 4, in [6]).

To show that the DP method for the singular source is also applicable for the high order finite difference scheme, we also solve the equation with the fifth order WENO finite difference scheme. The right figure of Figure 1 shows the pointwise errors in logarithmic scale with the WENO scheme using $N=64,100$ grid points at the final time $t=10.3$. For the comparison, the result with the Chebyshev collocation method using $N=16,32,64$ collocation points is also shown in the left figure of Figure 1. As shown in the figure, the DP method also works well with the WENO method and convergence near $x=0$ is also observed although it is slower than the other region.

\subsection{Nonlinear sine-Gordon equation}

Now we consider solving a non-linear scalar PDE with a stationary singular source, namely, the sine-Gordon equation. The sine-Gordon equation in a disordered media $[9,10]$ might 
Chebyshev collocation method

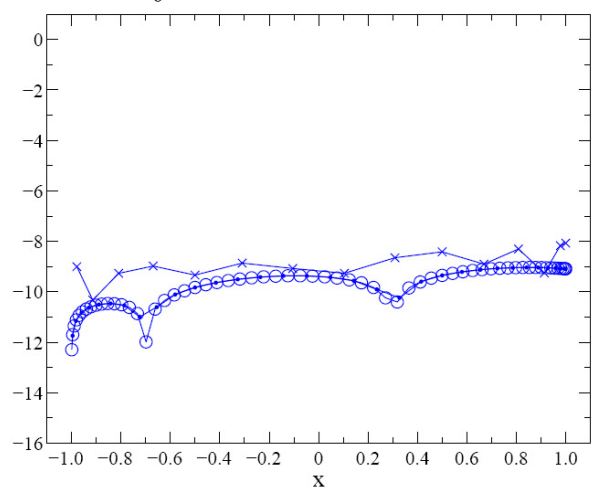

Fifth Order WENO scheme

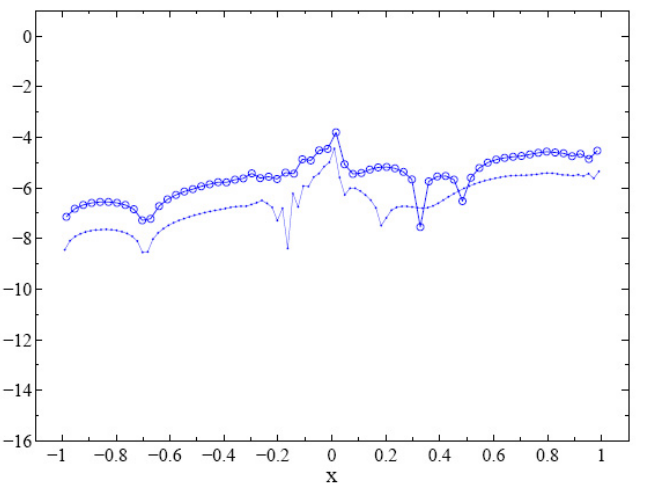

Figure 1: Pointwise error (in logarithm scale) of the solution for the linear wave equation (3.1) with a stationary source term at $t=10.3$. Left: the Chebyshev collocation method using $N=16,32,64$ Chebyshev-Lobatto collocation points. Right: with the fifth order WENO finite difference method using $N=64,100$. The DP method is applied to the approximation of $\delta$-function.

possesses a point-like defect. The defect is modeled as a $\delta$-function as a source term in the following equation

$$
u_{t t}-u_{x x}+\sin (u)=P(u), \quad-\infty<x<\infty, \quad t>0,
$$

where $P(u)$ is the potential term due to the defect and can be described as

$$
P(u)=\epsilon \delta(x) \sin (u) .
$$

The non-zero constant $\epsilon$ is the measure of the strength of the defect. If $\epsilon$ is zero, we obtain the regular sine-Gordon equation. If $\epsilon$ is large, the effect by the defect is also large to the global structure of the solution.

The governing equation (3.2) can be rewritten as

$$
u_{t t}-u_{x x}+(1-\epsilon \delta) \sin u=0 .
$$

This equation is not integrable due to the term involving the $\delta$-function if $\epsilon \neq 0$. However, ignoring the $\delta$-function source term, the sine-Gordon equation is integrable and there exists a soliton solution, known as the kink solution,

$$
u(x, t)=4 \tan ^{-1} \exp (\sigma z(x, t)),
$$

where

$$
z(x, t)=\frac{x-X}{\sqrt{1-V^{2}}}, \quad X=V t+X_{0},
$$

and $X$ is the kink coordinate, $V$ is the kink velocity and $\sigma= \pm 1$ is the kink polarity. $X_{0}$ is the initial location of the kink. 
Using the DP method, we will attempt to simulate the interaction of the kink solution of the sine-Gordon equation with a stationary singular source term. We performed the numerical study using the Chebyshev collocation method and the second order finite difference scheme in space $x \in[-8,8]$. The solution is advanced in time using the second order finite difference scheme.

The solution of the problem is defined by the boundary conditions at $x= \pm 8$. The incoming boundary condition is imposed at the left boundary

$$
u(-8, t)=\frac{-8-X}{\sqrt{1-V^{2}}}, \quad X=V t+X_{0},
$$

and the outflow boundary condition is imposed at the right boundary $x=8$, such that at the boundary point the PDE $u_{t}+u_{x}=0$ is satisfied, that is,

$$
u(8, t) \leftarrow u(8, t)-\frac{\Delta t}{\Delta x}(u(8, t)-u(8-\Delta x, t)) .
$$

With these boundary conditions, the problem models the nonlinear interaction between the $\delta$-like forcing term and the kink solution which is coming into the physical domain through the left boundary with the constant speed $V$. The global solution moves toward the right boundary and exits the physical domain at the right boundary. The singular potential term yields the so-called critical behavior of the solution. It has been discussed that there exists a critical velocity $V_{c}[9,10]$ such that

- if $V<V_{c}$, the kink solution $u(x, t)$ is trapped at the potential well.

- if $V>V_{c}$, the kink solution $u(x, t)$ will eventually penetrate the potential well.

Hence, when $V \sim V_{c}$, one can expect to observe the critical behavior of the solution. For more details about the critical behavior of the problem, we refer the readers to $[9,10]$.

Using the Chebyshev collocation method with the DP method for the singular source term, the semi-discretized version of the sine-Gordon equation can be written in the form of

$$
\frac{d^{2} U_{j}(t)}{d t^{2}}=\left.D_{N}^{2} U(x, t)\right|_{x=x_{j}}-\left.\left(I-\epsilon D_{N} H_{N}(x)\right)\right|_{x=x_{j}} \sin \left(U\left(x_{j}, t\right)\right) .
$$

A simple second order finite difference in time is used such that

$$
\frac{d^{2} U_{j}(t)}{d t^{2}} \rightarrow \frac{U_{j}^{n+1}-2 U_{j}^{n}+U_{j}^{n-1}}{\Delta t^{2}} .
$$

where $U_{j}^{n}=U\left(x_{j}, t_{n}\right), \quad n=2, \ldots$, with a time step $\Delta t$ and $t_{n}=n \Delta t$. The starting solution at the first time step $U_{j}^{1}$ can be computed via a high order multi-step method.

For the comparison, we also solved the problem with a second order finite difference method with the GA method for the $\delta$-function. For the numerical example, we use $\epsilon=0.5$ following [9]. 
It is quite surpising to see that the global collocation method works very well despite of the fact that the DP method uses highly oscillatory approximation (the Gibbs phenomenon) of the $\delta$-function on the collocation points. Through a series of experiments with differenct velocity $V$, the critical velocity $V_{c}$ was found to be $0.121582<V_{c}<0.121583$ with the Chebyshev collocation method using $N=128$ collocation points, and with the second order finite difference method using $N=2000$ uniformly spaced grid points.

Finite difference scheme

Chebyshev collocation method
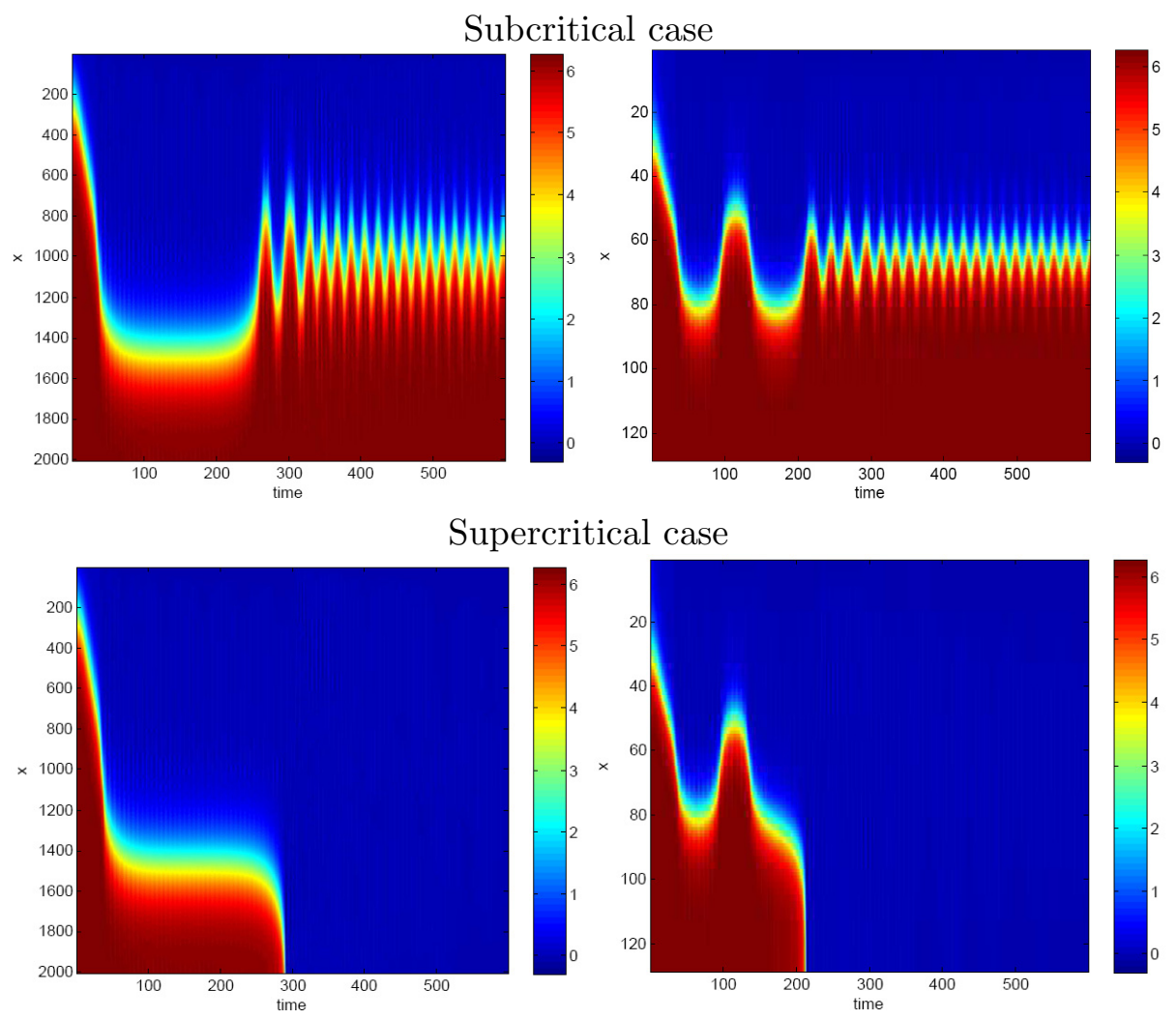

Figure 2: (Top) Subcritical and (Bottom) Supercritical behaviors of the sine-Gordon equation with stationary singular source. Left: the second order finite difference method with $N=2000$ grid points. Right: the Chebyshev collocation methods with $N=128$ collocation points.

The top two figures of Figure 2 shows the solution behaviors at the sub-critical velocity $V<V_{c}$ for both methods. The solutions are similiar but the results with the Chebyshev collocation method clearly shows the one-bounce solution while the solution computed by the finite difference method monotonically exits the domain. For the super-critical case $V>V_{c}$, results from both methods show that the kink solution eventually penetrates the potential well and exits the domain (see the bottom two figures of Figure 2). The exit times are, however, different and the global behaviors are also different for both methods. The GA method uses the local approach to the approximation of the $\delta$-function while the DP method uses the global apporach. The DP method is a more consistent way of 
approximating the $\delta$-function. Our numerical experiments show that the global structures of the solutions with the GA and DP methods remain the same as the grids are refined. More detailed study will be given in our future work [7].

\section{Linear scalar PDEs with a non-stationary singular source}

In this section, we will study the effect of the non-stationary singular source on the solution of hyperbolic equations. The linear scalar wave equation is employed as the model of this problem. This particular case illustrates some perils of modeling the singular source which is moving with a non-zero speed inside the computational domain.

Consider the following simple scalar one-way wave equation

$$
u_{t}+u_{x}=\epsilon \delta(x+c t), \quad x \in[-1,1], \quad t>0,
$$

where $c>0$ is the speed of moving $\delta$-function and $\epsilon=1$. For simplicity, we assume that $c$ is a constant with time. Together with the given initial and boundary conditions

$$
u(x, 0)=\sin (\pi x), \quad u(-1, t)=\sin (\pi(-1-t)),
$$

the exact solution of this problem is

$$
u(x, t)=\left\{\begin{array}{ll}
\sin (\pi(x-t)), & x<-c t \\
\sin (\pi(x-t))+H(x+c t), & x \geq-c t
\end{array} .\right.
$$

The equation (4.1) is solved with the fifth order WENO-Z finite difference scheme. We will use $c=1 / 16$ and refine the grid by doubling the number of grid points $N=250,500,1000$. The scheme is advanced to the final time $t=10$ using the third order TVD Runge-Kutta scheme with the CFL number, $C F L=0.01$ or smaller.
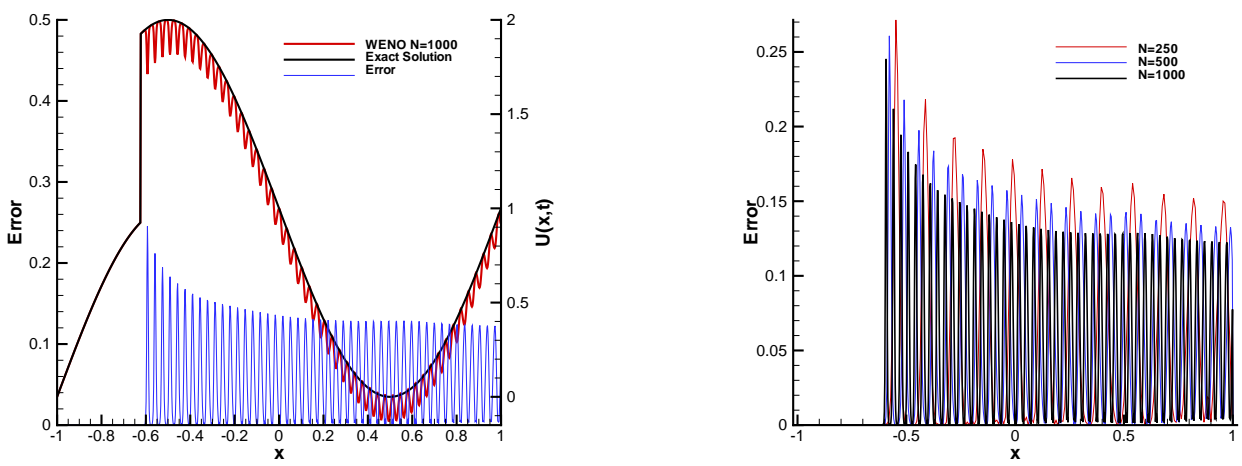

Figure 3: Left: the exact solution (black), numerical solution (red) and the pointwise error (blue) of the scalar wave equation with a non-stationary singular source, which is being approximated by the DP method, at $t=10$ using $N=1000$ grid points. Right: the pointwise error of the solution at $t=10$ with $N=250,500,1000$. 
In the left figure of Figure 3, the exact and the numerical solution of the problem with the non-stationary singular source, which is being approximated by the DP method, are shown at $t=10$ with $N=1000$ grid points. The singular source term has moved from $x=0$ to $x=-5 / 8$. Here we see that the numerical solution yields a small scale oscillations to the right of the singular source term. It should be noted that similar small scale oscillations (albeit obscured by the Gibbs oscillations) can also be observed if one simulates the same problem with the spectral method. Since the high order WENO finite difference schemes are often used for capturing solutions exhibiting singular behaviors like shock solutions, one would lead to believe that the oscillations could be real. To demonstrate more clearly that the oscillations are not real, we use the simple frequency analysis. It is found that the frequency of the oscillations is directly proportional to the number of grid points $N$. In the right figure of Figure 3, it is shown that the frequency of the oscillations is doubled as the number of grid points $N$ is doubled. Furthermore, the amplitude of the oscillations does not decrease much even when the grid is highly refined. In other words, these oscillations are purely numerical artifact due to the discretization of the $\delta$-function on the discrete finite grid. As the $\delta$-function moves, the discrete grid can no longer support the definition of the $\delta$-function between the two grid points resulting in the oscillatory behavior. In this particular case, we measure the wavelength of the oscillations from the data and find that the wavelength $\lambda_{N}=0.137,0.0675,0.0339$ for $N=250,500,1000$, respectively. The reason for this is as following. The grid spacing is $\Delta x$ and the speed of propagation of the $\delta$-function is $c$. The given PDE will only convey the full effect of the $\delta$-function only when $\delta$-function jumps from one grid point $x_{j}$ to the next grid point $x_{j+1}$. The time interval that $\delta$-function takes to make the full jump will be $\Delta t=\lambda_{N}=\Delta x / c=2 /(N c)$, which yields the corresponding values of $\lambda_{N}=0.128,0.064,0.032$. This is why the frequency of the oscillations is doubled when the number of grid points is doubled. In the case of the stationary singular source, $c$ tends to zero. Then the wavelength of the oscillations tends to become $\infty$. This explains why the numerical oscillations will not appear in the stationary singular source.
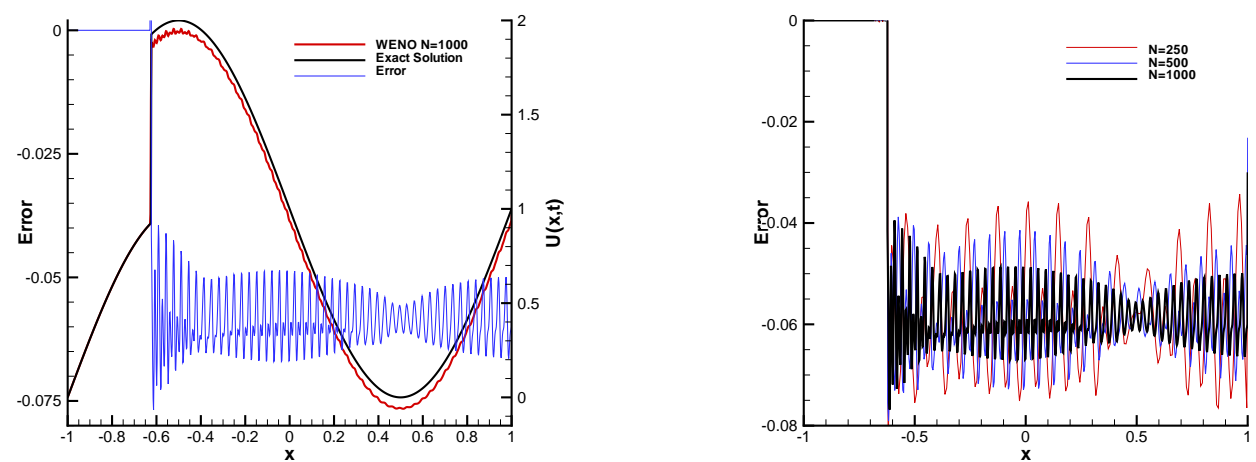

Figure 4: (Left) The exact solution (black), numerical solution (red) and the error (blue) of the scalar wave equation with a non-stationary singular source, which is being approximated by the GA method, at $t=10$ using with $N=1000$ grid points. (Right) The pointwise error of the solution at $t=10$ with $N=250,500,1000$. 
In Figure 4, similar small scale oscillations appear in the solution of the problem with the non-stationary singular source, which is being approximated by the GA method, at $t=10$ using $N=1000$ grid points. The pointwise error of the solution is also shown with $N=250,500,1000$ grid points in the right figure.

Similar results have been obtained for different version of the WENO finite difference methods, different CFL number and different speed of the moving $\delta$-function, $c$.

\section{Conclusion}

In this paper, we consider the linear and nonlinear scalar PDEs for both the stationary and non-stationary singular source term represented by the Dirac $\delta$-function. We use the Gaussian approximation method and the direct projection method for the approximation of the $\delta$-function. It has been shown that the direct projection method yields a good result for the nonlinear problems as well. For the nonlinear problem, we solve the sine-Gordon equation with the point-limit defect modeled by the $\delta$-function. Compared to the results with the finite difference method, the spectral approximation with the direct projection method reveals the details of dynamics of the solution with great efficiency.

We also study the numerical issues arising when the non-stationary source term is considered for the linear wave equation as the model problem for general hyperbolic PDEs with a non-stationary singular source term. We solve numerically such model problem on a discrete grid system using the spectral collocation and the high order WENO-Z finite difference scheme. It is shown that the uncertainty of the location of the $\delta$-function between grid points causes the numerical oscillations. Since the $\delta$-function is propagating through the discrete grid points, the oscillations are characterized as the periodic bump solutions when the $\delta$-function passes through the grid points. Such oscillations are propagating over the domain according to the given PDE. If the magnitude of the singular source term is small, the oscillations would be small. They are, however, inevitable as long as the $\delta$-function is approximated on the discrete grid points. We show the relation of the frequency of the oscillations to the step size of the grids and speed of the propagation of the $\delta$-function. It is shown that the frequency of oscillations is doubled if the number of grid points is doubled. Such oscillations albeit small should be addressed carefully when the non-stationary singular source term is involved in hyperbolic PDEs.

In our future work, we will consider more general type of PDEs with the direct projection method and extend the current method to higher dimensional problems. Our future work will also center around the development of the accurate and stable direct projection method for the non-stationary $\delta$-function.

\section{Acknowledgements}

The first author (Jung) acknowledges the support of this work from the NSF under Grant No. DMS-0608844. The second author (Don) would like to thank the support provided by the FRG grant from Hong Kong Baptist University and partial funding support from AFOSR YIP grant of Professor Gustaaf Jacobs at San Diego State University. 


\section{$\underline{\text { References }}$}

[1] R. Borges, M. Carmona, B. Costa \& W. S. Don, An Improved Weighted Essentially Non-Oscillatory Scheme for Hyperbolic Conservation Laws, J. Comput. Phys. 227 pp. 3191-3211, 2008

[2] B. Engquist, A.-K. Tornberg \& R. Tsai, Discretization of Dirac delta function in level set methods, J. Comput. Phys. 207, pp. 28-51, 2005.

[3] D. Gottlieb \& A. Orzag, Numerical Analysis of Spectral Methods: Theory and Applications, SIAM, Philadelphia, 1977

[4] J. Hesthaven, S. Gottlieb \& D. Gottlieb, Spectral methods for time-dependent problems, Cambridge UP, Cambridge, UK, 2007.

[5] G. Jacobs \& W. S. Don, A high-order WENO-Z finite difference based particle-sourcein-cell method for computation of particle-laden flows with shocks, J. Comput. Phys. 228 pp. $1365-1379,2008$

[6] J.-H. Jung, A Note on the Spectral Collocation Approximation of Some Differential Equations with Singular Source Terms, J. Sci. Comput. 39 (1) pp. 1573-7691, 2009.

[7] J.-H. Jung, A spectral collocation approximation of nonlinear interactions of solitons in disordered media, in preparation.

[8] J.-H. Jung, G. Khanna \& I. Nagle, A spectral collocation approximation of onedimensional head-on collisions of black holes, arXiv:gr-qc/0711.2545.

[9] Z. Fei, Y. S. Kivshar \& L. Vázquez, Resonant kink-impurity interactions in the sineGordon model, Phy. Rev. A 45 (8), pp. 6019-6030, 1992.

[10] R. H. Goodman \& R. Haberman, Chaotic scattering and the $n$-bounce resonance in solitary-wave interactions, Phy. Rev. Lett. 98 (10), pp. 104103-1-104103-4, 2007.

[11] C. S. Peskin, The immersed boundary method, Acta Numer. 11, pp.479-517, 2002.

[12] A.-L. Tornberg \& B. Engquist, Numerical approximations of singular terms in differential equations, J. Comput. Phys. 200, pp. 462-488, 2004.

[13] C.-W. Shu, High order weighted essentially non-oscillatory schemes for convection dominated problems, SIAM Review 51, pp.82-126, 2009.

[14] J. Waldén, On the approximation of singular source terms in differential equations, Numer. Methods Partial Diff. Eqs. 15, pp. 503-520, 1999. 